\title{
WEAKLY ORDERED A-COMMUTATIVE PARTIAL GROUPS OF LINEAR OPERATORS DENSELY DEFINED ON HILBERT SPACE
}

\author{
JIŘÍ JANDA* \\ Department of Mathematics and Statistics, Faculty of Science, Masaryk University, Kotlářská 2, CZ-611 37 Brno, \\ Czech Republic \\ * corresponding author: 98599@mail.muni.cz
}

\begin{abstract}
The notion of a generalized effect algebra is presented as a generalization of effect algebra for an algebraic description of the structure of the set of all positive linear operators densely defined on a Hilbert space with the usual sum of operators. The structure of the set of not only positive linear operators can be described with the notion of a weakly ordered partial commutative group (wop-group). Due to the non-constructive algebraic nature of the wop-group we introduce its stronger version called a weakly ordered partial a-commutative group (woa-group). We show that it also describes the structure of not only positive linear operators.
\end{abstract}

KEYWORDS: (generalized) effect algebra, partial group, weakly ordered partial group, Hilbert space, unbounded linear operator, self-adjoint linear operator.

AMS Mathematics Subject Classification: 06F05, (03G25, 81P10, 08A55).

\section{INTRODUCTION}

The notion of an effect algebra was presented by Foulis and Bennett in 3 . The definition was motivated by giving an algebraic description of positive self-adjoint linear operators between the zero and the identity operator in a complex Hilbert space $\mathcal{H}$. The notion of a generalized effect algebra extends these ideas on unbounded sets of positive linear operators. To answer the natural question concerning the structure of sets of not only positive linear operators Paseka started to investigate a partially ordered commutative group of operators with a fixed domain [5]. In [6] Paseka and Janda introduced the structure of a weakly ordered partial commutative group (shortly a wop-group). They also showed that the set of all linear operators on complex Hilbert space $\mathcal{H}$ with the usual sum, which is restricted to the same domain for unbounded operators (partial operation $\oplus_{\mathcal{D}}$ ), possesses this structure. In [4] we considered the structure on the important subset of self-adjoint operators, showing that it is also a wop-group.

Wop-groups have only a non-constructive associativity (the equation holds if and only if both sides are defined). It has been shown [4 that the set of all linear operators has generally stronger algebraic properties. This was a motivation for introducing the notion of a weakly ordered partial a-commutative group (woa-group) where the associative law is more constructive. Also a weak order is more strongly related to the partial operation. Moreover, every positive cone of a woa-group is a generalized effect algebra. On the other hand, we present a construction showing that every generalized effect algebra is a positive cone of some woa-group.

\section{Preliminaries}

We review some basic terminology, definitions and statements. The basic reference for this text is the book by Dvurečenskij and Pulmannová [2].

Definition 1. A partial algebra $(E,+, 0)$ is called a generalized effect algebra if $0 \in E$ is a distinguished element and + is a partially defined binary operation on $E$ which satisfies the following conditions for any $x, y, z \in E$ :

(GEi) $x+y=y+x$, if one side is defined,

(GEii) $(x+y)+z=x+(y+z)$, if one side is defined,

(GEiii) $x+0=x$,

(GEiv) $x+y=x+z$ implies $y=z$ (cancellation law),

(GEv) $x+y=0$ implies $x=y=0$.

In every generalized effect algebra $E$ the partial binary operation - and relation $\leq$ can be defined by

(ED) $x \leq y$ and $y-x=z$ iff $x+z$ is defined and $x+z=y$.

Then $\leq$ is a partial order on $E$ under which 0 is the least element of $E$. A generalized effect algebra with the top element $1 \in E$ is called an effect algebra and we usually write $(E,+, 0,1)$.

A subset $S$ of $E$ is called a sub-generalized effect algebra (sub-effect algebra) of $E$ iff (i) $0 \in S(1 \in S)$, (ii) if out of elements $x, y, z \in E$ such that $x+y=z$ at least two are in $S$, then all $x, y, z \in S$.

Definition 2. 6 A partial algebra $(G,+, 0)$ is called a commutative partial group if $0 \in E$ is a distinguished 
element and + is a partially defined binary operation on $E$ which satisfies the following conditions for any $x, y, z \in E$ :

(GPi) $x+y=y+x$ if $x+y$ is defined,

(GPii) $(x+y)+z=x+(y+z)$ if both sides are defined,

(GPiii) $x+0$ is defined and $x+0=x$,

(GPiv) for every $x \in E$ there exists a unique $y \in E$ such that $x+y=0$ (we put $-x=y$ ),

$(\mathrm{GPv}) x+y=x+z$ implies $y=z$ (cancellation law).

We say that a commutative partial group $(G,+, 0)$ is weakly ordered (shortly a wop-group) with respect to a reflexive and antisymmetric relation $\leq$ on $G$ if $\leq$ is compatible w.r.t. partial addition, i.e., for all $x, y, z \in G, x \leq y$ and both $x+z$ and $y+z$ are defined implies $x+z \leq y+z$.

Due to the non-constructive algebraic nature of wop-groups, we will introduce a stronger structure with the notion of a woa-group.

Definition 3. A partial algebra $(G,+, 0)$ is called an a-commutative partial group if $0 \in G$ is a distinguished element and + is a partially defined binary operation on $G$ which satisfies the following conditions for any $x, y, z \in G$ :

(Gi) $x+y=y+x$ if $x+y$ is defined,

(Gii) $x+0$ is defined and $x+0=x$,

(Giii) for every $x \in E$ there exists a unique $y \in E$ such that $x+y=0$ (we put $-x=y$ ),

(Giv) If $(x+y)+z$ and $(y+z)$ are defined, then $x+(y+z)$ is defined and $(x+y)+z=x+(y+z)$.

An a-commutative partial group $(G,+, 0)$ is called weakly ordered (shortly a woa-group) with respect to a reflexive and antisymmetric relation $\leq$ on $G$ (we call it a weak order) if

(Ri) $x \leq y$ iff there exists $0 \leq z, x+z$ is defined and $x+z=y$,

(Rii) $0 \leq x, y$ and $x+y$ defined, then $0 \leq x+y$,

(Riii) $0 \leq x, 0 \leq z, x \leq y$ and $y+z$ defined implies $x+z$ defined.

Note that in the case of generalized effect algebras the partial order is induced from a partial operation. On the other hand, the weak order for woa-groups (wop-groups) can be chosen in various ways. We will show that the weak order is determined by the partial operation and the set of positive elements.

Remark 1. Using the commutativity (Gi) with the axiom (Giv), we can obtain similar formulas to (Giv) for any permutation of variables. That is, let us have an a-commutative group $(G, 0,+)$. Then for any $x, y, z \in G$, from the existence of $(x+y)+z$ and $x+z$ we have $(x+y)+z=(x+z)+y$. Or similarly, the existence of $x+(y+z)$ and $x+y$ implies $x+(y+z)=(x+y)+z$ and so on. In the following, we will be using (Giv) in this more general sense and we omit mentioning the commutativity.

Definition 4. Let $(G,+, 0)$ be an (a-)commutative partial group and let $S$ be a subset of $G$ such as

(Si) $0 \in S$,

(Sii) $-x \in S$ for all $x \in S$,

(Siii) for every $x, y \in S$ such that $x+y$ is defined also $x+y \in S$.

Then we call $S$ an (a-)commutative partial subgroup of $G$.

Let $G$ be a wop-group (woa-group) with respect to a relation $\leq^{G}$ and let $\leq^{S}$ be a relation on a (a-)commutative partial subgroup $S \subseteq G$. If for all $x, y \in S$ holds: $x \leq^{S} y$ if and only if $x \leq^{G} y$, we call $S$ a wop-subgroup (woa-subgroup) of $G$.

Lemma 1. Let $(G,+, 0)$ be an a-commutative partial group. Then for any $a, b, c, x, y, z \in G$ the following holds:

(1.) $a+c=b$ iff $c=b+(-a)$,

(2.) $a+x=(a+y)+z$ implies $x=(y+z)$,

(3.) whenever $a+b$ is defined, then $(-a)+(-b)$ is defined and $(-a)+(-b)=-(a+b)$.

Proof. (1.) We have $c=c+(a+(-a))=(c+a)+$ $(-a)=b+(-a)$.

(2.) Let us have $a+x=(a+y)+z$. Then $x=$ $((a+y)+z)+(-a)$. Since $y+a$ and $y+(a+(-a))$ are defined then $(y+a)+(-a)$ is defined. We have $x=((a+y)+z)+(-a)=(((a+y)+(-a))+z)=y+z$.

(3.) Let us have $a, b \in G$ such that $a+b \in G$. We have $a=a+(b+(-b))=(a+b)+(-b)$ defined. Then by (1.) $(-b)=a+(-(a+b))$ and also by (1.) $(-a)+(-b)=(-(a+b))$.

Lemma 2. Let $(G,+, 0)$ w.r.t. $\leq$ be a woa-group. Then for any $a, b, c, x, y, z \in G$ the following holds:

(1.) $a \leq b$ iff $b+(-a) \geq 0$,

(2.) $a \leq b$ iff $-b \leq-a$.

Proof. (1.) Let $a \leq b$. Then there exists $c \geq 0$ such that $a+c=b$ and from Lemma 1 (1.) $b+(-a) \geq 0$. On the other hand, let $b+(-a) \geq 0$. Then $b=$ $b+(a+(-a))=a+(b+(-a))$ hence by $(\mathrm{Ri}) a \leq b$.

(2.) Let $a \leq b$ for some $a, b \in G$. Then $(b+(-a)) \geq 0$ and $-a=((-b)+b)+(-a)=(b+(-a))+(-b)$ ie. $-b \leq-a$.

Lemma 3. Every a-commutative partial group $(G,+, 0)$ is a partial commutative group.

Proof. The cancellation law follows from Lemma 1 (2.) choosing $z=0$. 
Lemma 4. Every woa-group $(G,+, 0)$ w.r.t. $\leq$ is a wop-group w.r.t. $\leq$.

Proof. By the previous lemma, we have $(G,+, 0)$ is a partial commutative group. Let us have $x, y, z \in G$, $x \leq y, x+z, y+z$ defined. Then by Lemma 2 (1.) $0 \leq(y+(-x))$ and $x+(y+(-x))=y$ hence $y+z=(x+(y+(-x))+z=(x+z)+(y+(-x))$ and according to (Rii) $x+z \leq y+z$.

Example 1. Let $G=\{0, a, b, c,-a,-b,-c\}$ be a set with partial operation + defined for $a+b=c$ and $0+x=x, x+(-x)=0$ for all $x \in G$. Then $(G,+, 0)$ is a commutative partial group, but it is not an a-commutative partial group. Note that even $c+(-b)=(a+b)+(-b)$ is not defined.

Lemma 5. Let $(G,+, 0)$ be an a-commutative partial group and $S \subseteq G$ its a-commutative partial subgroup. Then $\left(S,+{ }_{S}, 0\right)$ is an a-commutative partial group.

Proof. Immediately (Gi) and (Giv) follows from (Siii), (Gii) from (Si), (Giii) from (Sii).

Lemma 6. Let $(G,+, 0)$ be a woa-group w.r.t. $\leq$ and $S \subseteq G$ its woa-subgroup w.r.t. $\leq S$. Then $(S,+/ S, 0)$ w.r.t. $\leq^{S}$ is a woa-group.

Proof. By the previous lemma $(S,+/ S, 0)$ is an acommutative partial group. For (Ri) let $x, y \in S$, $x \leq^{S} y$. Then by Lemma 2 (1.) $0 \leq y+(-x)$ is defined and by (Siii) $y+(-x) \in S$. The other direction is straightforward. (Rii) is clear and (Riii) follows from (Siii).

Corollary 1. Let $(G,+, 0)$ be a wop-group w.r.t. $\leq$ and $S \subseteq G$ its wop-subgroup w.r.t. $\leq S$. Whenever $(G,+, 0)$ w.r.t. $\leq$ is also a woa-group, then $S$ is its woa-subgroup.

Theorem 1. Let $(G,+, 0)$ w.r.t. $\leq$ be a woa-group. Then the set $\operatorname{Pos}(G)=\{x \in G \mid 0 \leq x\}$ with the restriction of the partial operation + on $\operatorname{Pos}(G)$, i.e., $(\operatorname{Pos}(G),+/ \operatorname{Pos}(G), 0)$ forms a generalized effect algebra.

Proof. (GEi) and (GEiii) hold from definition and the cancellation law follows from Lemma 4 . For the axiom (GEv) let $x, y \in \operatorname{Pos}(G)$ such that $x+y=0$. Then $x=(-y)$ and with Lemma 2 (2.) we have $-y \leq 0$ hence $x=y=0$. We will verify (GEii). Let us have $x, y, z \in \operatorname{Pos}(G)$ such that $(x+y)+z$ is defined. Therefore $y \leq x+y,(x+y)+z$ exists and (Riii) implies that $y+z$ exists. Using (Giv) we have $(x+y)+z=x+(y+z)$.

Lemma 7. Let $(G,+, 0)$ be an a-commutative partial group and $E \subseteq G$ a subset closed under the + , i.e., $x, y \in E, x+y \in G$ implies $x+y \in E$, such that $0 \in E$ and $\left(E,+/_{E}, 0\right)$ forms a generalized effect algebra. Define a relation $\leq$ by $x \leq y$ iff $(-x)+y$ is defined and $((-x)+y) \in E$. Then $(G,+, 0)$ is a woa-group w.r.t. $\leq, \operatorname{Pos}(G)=E$ and $\leq$ on $\operatorname{Pos}(G)$ coincides with induced partial order $\leq^{E}$ from $(E,+/ E, 0)$.
Proof. Reflexivity is clear since $0 \in E$. Let $x \leq y$ and $y \leq x$, then $-x+y, x+(-y) \in E$ and with (Giv) $(-x+x)+(-y+y)=(-x+y)+(x+(-y))=0$. $E$ is a generalized effect algebra hence by (GEv) $x+(-y)=-x+y=0$ that is $x=y$. Clearly $\operatorname{Pos}(G)=E$. Then ( $\mathrm{Ri})$ is straightforward using the definition of $\leq$ and Lemma 1 (1.). (Rii) holds because we want $E$ to be closed under the + . For (Riii), let $x, z \in E, x \leq y$ and $y+z$ be defined. Then $y+z=(x+((-x)+y))+z=(x+z)+((-x)+y))$ using the associativity of generalized effect algebra $E$ since $x, z,((-x)+y) \in E$.

We show the coincidence. Let us have $-x+y, x, y \in$ $E$. Because $E$ is closed on + we have $x+(-x+y)$ defined and $x+(-x+y)=y$, i.e., $x \leq^{E} y$. On the other hand let $x \leq^{E} y, x, y \in E$. Then there exists $z \in E$ such that $x+z=y$ and by Lemma 1 (1.) $z=y+(-x)$.

The previous lemma formalizes the idea of determining a weak order by the set of positive elements. Let us have an a-commutative partial group and choose some elements to be positive. Consider the smallest set closed under partial addition containing zero and the chosen elements. If the set has the form of a generalized effect algebra, then there exists such a weak order that our set is exactly the set of positive elements. On the other hand, it is not hard to show that if the set is not a generalized effect algebra, then there is no such weak order that all of our chosen elements are positive.

Theorem 2. Let $(E,+, 0)$ be a generalized effect algebra with induced order $\leq$. Then there exists a woa-group $(G, \oplus, 0)$ w.r.t. relation $\leq^{G}$ such that $(\operatorname{Pos}(G), \oplus / \operatorname{Pos}(G), 0)=(E,+, 0)$ and $\leq_{/ \operatorname{Pos}(G)}^{G}=\leq$.

Proof. Let $(E,+, 0)$ be a generalized effect algebra with induced order $\leq$. For any $a, b \in E, a \leq b$, the symbol $b-a$ denotes such an element that $a+(b-a)=b$. Let $E^{-}$be a set with the same cardinality disjoint from $E$.

Consider a bijection $\varphi: E \rightarrow E^{-}$. We set $a^{-}=\varphi(a)$ for $a \in E \backslash\{0\}$ and $0^{-}=0$. Let

$$
G=E \dot{\cup}\left(E^{-} \backslash\{\varphi(0)\}\right)
$$

be a disjoint union of $E$ and $E^{-} \backslash\{\varphi(0)\}$.

Let us define define a partial binary operation $\oplus$ on $G$ by

- $a \oplus b$ exists iff $a+b$ exists and then $a \oplus b=a+b$ for all $a, b \in E$,

- $a^{-} \oplus b^{-}$exist iff $a+b$ exists and then $a^{-} \oplus b^{-}=$ $(a+b)^{-}$for all $a, b \in E$,

- $a \oplus b^{-}=b^{-} \oplus a$ is defined iff

(1.) $b \leq a(a-b$ exists $)$ then $a \oplus b^{-}=a-b$ or

(2.) $a \leq b(b-a$ exists $)$ then $a \oplus b^{-}=(b-a)^{-}$for any nonzero $a, b \in E$. 
It is not hard to show that the definition is correct. For any $a, b \in E$ it holds that

$\left(\alpha_{1}\right)(a \oplus b)^{-}=(a+b)^{-}=\left(a^{-} \oplus b^{-}\right)$.

Let $a \oplus b^{-}$be defined and

$$
\left(\beta_{1}\right) b \leq a(a-b \text { exists }) \text {, then }\left(a \oplus b^{-}\right)^{-}=(a-b)^{-}=
$$
$a^{-} \oplus b$.

We show that $(G, \oplus, 0)$ forms an a-commutative partial group. Commutativity $(\mathrm{Gi})$ is clear from the definition. Since $0 \in E$ it follows $a \oplus 0=(a+0)=a$ and $\left(a^{-} \oplus 0\right)=(a-0)^{-}=a^{-}$for all $a \in E$, that is (Gii). Clearly for any $a \in E$ there exists $a^{-} \in E^{-}$ where $a \oplus a^{-}=a-a=0$. This defines also inverse elements for any $a^{-} \in E^{-}$.

Let us verify the associativity case by case. We assume that $x, y, z \in E$.

Case $i$. First, let us have $(x \oplus y) \oplus z$ defined and $y \oplus z$ defined. Then $(x \oplus y) \oplus z=(x+y)+z=x+(y+z)=$ $x \oplus(y \oplus z)$ where the existence and the equation follow from the associativity of the generalized effect algebra E.

Case ii. Let $(x \oplus y) \oplus z^{-}$and $y \oplus z^{-}$be defined. And let

$\left(\alpha_{2}\right) z \leq y$ (i.e., $y-z$ is defined). Hence $z \leq(x+y)$ and $(x \oplus y) \oplus z^{-}=(x+y)-z$. Since $y-z$ exists, we have $y=(y-z)+z$ and then $((x+y)-z)+$ $z=x+y=x+((y-z)+z)=(x+(y-z))+z$, where we used the associativity of the generalized effect algebra $E$. By the cancellation law we have $(x \oplus y) \oplus z^{-}=(x+y)-z=x+(y-z)=x \oplus\left(y \oplus z^{-}\right)$.

$\left(\beta_{2}\right) y \leq z$ and $z \leq(x+y)$ (that is $z-y$ and $(x+y)-z$ exist $)$. We have $z=(z-y)+y$ and also $((x+y)-z)+z=x+y$. Putting together $((z-y)+y)+((x+y)-z)=x+y$ from which $(z-y)+((x+y)-z)=x$ hence $(x+y)-z=x-(z-y)$. So we have $(x \oplus y) \oplus z^{-}=(x+y)-z=x-(z-y)=$ $x \oplus\left(z \oplus y^{-}\right)^{-}=x \oplus\left(z^{-} \oplus y\right)$. The last equation holds by $\beta_{1}$.

$\left(\gamma_{2}\right) y \leq z$ and $(x+y) \leq z$ (hence $z-y$ and $z-(x+y)$ exist). From $(z-y)+y=(z-(x+y))+(x+y)$ we have $z-y=(z-(x+y))+x$ hence $(z-y)-x=z-(x+y)$. Therefore $(x \oplus y) \oplus z^{-}=(z-(x+y))^{-}=((z-y)-$ $x)^{-}=\left(x \oplus(z-y)^{-}\right)=x \oplus\left(y \oplus z^{-}\right)$.

Case iii. Let $\left(x^{-} \oplus y\right) \oplus z$ and $y \oplus z$ be defined. Let

$\left(\alpha_{3}\right) x \leq y$ (i.e., $y-x$ is defined and also $x \leq y \leq$ $y+z)$. Then $y=(y-x)+x$ and $y+z=((y-x)+x)+z$ hence $(y+z)-x=(y-x)+z$. Therefore $\left(x^{-} \oplus y\right) \oplus z=$ $(y-x)+z=(y+z)-x=x^{-} \oplus(y \oplus z)$.

$\left(\beta_{3}\right) y \leq x$ and $(x-y) \leq z$ (that is $x-y$ and $z-(x-y)$ are defined). Since $z+y$ is defined, we have from $z=(z-(x-y))+(x-y)$ with $x=(x-y)+y$ the equation $z+y=(z-(x-y))+x$ and $(z+y)-x=$ $z-(x-y)$. Hence $\left(x^{-} \oplus y\right) \oplus z=(x-y)^{-} \oplus z=$ $z-(x-y)=(z+y)-x=x^{-} \oplus(y \oplus z)$.

$\left(\gamma_{3}\right) y \leq x$ and $z \leq(x-y)$ (hence $x-y$ and $(x-y)-z$ are defined). We have $(x-y)-z=$ $(x-y)-z$ from which $x=((x-y)-z)+y)+z$ hence $x-(y+z)=(x-y)-z$. And then $\left(x^{-} \oplus y\right) \oplus z=$ $(x-y)^{-} \oplus z=((x-y)-z)^{-}=(x-(y+z))^{-}=$ $\left(x \oplus(y \oplus z)^{-}\right)^{-}=x^{-} \oplus(y \oplus z)$ (the last equation follows from $\beta_{1}$ ).

Case iv. Let $\left(x \oplus y^{-}\right) \oplus z$ and $y^{-} \oplus z$ be defined. Let $\left(\alpha_{4}\right) y \leq x$ and $y \leq z$ (i.e., $x-y$ and $z-y$ are defined). Using $y=x-(x-y)$ and $z=(z-y)+y$ we get $(x-y)+z=(x-y)+((z-y)+y)=(z-y)+x$. Hence $\left(x \oplus y^{-}\right) \oplus z=(x-y)+z=(z-y)+x=x \oplus\left(y^{-} \oplus z\right)$.

$\left(\beta_{4}\right) y \leq x$ and $z \leq y$ (hence $x-y$ and $y-z$ are defined). Similarly $y=(y-z)+z$ and $x=(x-y)+y$ and then $x=(x-y)+((y-z)+z)$ from which $x-(y-z)=(x-y)+z$. Hence $\left(x \oplus y^{-}\right) \oplus z=$ $(x-y)+z=x-(y-z)=x-\left(y \oplus z^{-}\right)=x \oplus\left(y \oplus z^{-}\right)^{-}=$ $x \oplus\left(y^{-} \oplus z\right)$ (the last equation follows from $\left.\beta_{1}\right)$.

$\left(\gamma_{4}\right) x \leq y$ and $y \leq z$ which implies $(y-x) \leq z$ (hence $y-x, z-(y-x)$ and $z-y$ are defined). Then $z=(z-y)+y=(z-y)+((y-x)+x)$ from which $z-(y-x)=(z-y)+x$ and hence $\left(x \oplus y^{-}\right) \oplus z=$ $(y-x)^{-} \oplus z=z-(y-x)=(z-y)+x=x \oplus\left(y^{-} \oplus z\right)$.

$\left(\delta_{4}\right) x \leq y,(y-x) \leq z$ and $z \leq y$ (i.e., $y-x$, $z-(y-x)$ and $y-z$ are defined). Then $y=(y-z)+z=$ $(y-z)+((z-(y-x))+(y-x))=(y-x)+x$ hence $(y-z)+(z-(y-x))=x$ from which $\left(x \oplus y^{-}\right) \oplus z=$ $(y-x)^{-} \oplus z=z-(y-x)=x-(y-z)=x-\left(y \oplus z^{-}\right)=$ $x \oplus\left(y^{-} \oplus z\right)$ (using $\left.\beta_{1}\right)$.

$\left(\epsilon_{4}\right) x \leq y, z \leq(y-x)$ and $z \leq y$ (that is $y-x$, $(y-x)-z$ and $y-z$ are defined). Then $y=(y-x)+x=$ $((y-x)-z)+z)+x=(y-z)+z$. Hence $(y-x)-z)+x=$ $(y-z)$ and $\left(x \oplus y^{-}\right) \oplus z=(y-x)^{-} \oplus z=((y-x)-z)^{-}=$ $((y-z)-x)^{-}=\left(x \oplus(y-z)^{-}\right)=x \oplus\left(y^{-} \oplus z\right)$.

Until now, the map ${ }^{-}: E \rightarrow E^{-} \cup 0$ has been defined only for elements of $E$. We can extend it on $G$ by $\left(a^{-}\right)^{-}=a$, which gives us an involution on $G$. Then for any $a, b \in E$ whenever $b-a$ is defined it holds $\left(a \oplus b^{-}\right)^{-}=\left((b-a)^{-}\right)^{-}=(b-a)=a^{-} \oplus b$. Together with $\left(\alpha_{1}\right)$ and $\left(\beta_{1}\right)$ we have

$\left(\gamma_{1}\right)\left(a \oplus b^{-}\right)^{-}=a^{-} \oplus b$ for all $a, b \in E$,

$\left(\delta_{1}\right)\left(a^{-} \oplus b^{-}\right)^{-}=\left((a \oplus b)^{-}\right)^{-}=a \oplus b$ for all $a, b \in E$

Case v. Let $\left(x^{-} \oplus y^{-}\right) \oplus z$ and $y^{-} \oplus z$ be defined. Then also $y \oplus z^{-}$is defined and using $\left(\alpha_{1}\right),\left(\gamma_{1}\right)$ and (ii) we get $\left(x^{-} \oplus y^{-}\right) \oplus z=\left((x \oplus y) \oplus z^{-}\right)^{-}=\left(x \oplus\left(y \oplus z^{-}\right)\right)^{-}=$ $\left(x \oplus\left(y^{-} \oplus z\right)\right)$.

Case vi. Let $\left(x \oplus y^{-}\right) \oplus z^{-}$and $y^{-} \oplus z^{-}$be defined. Then $y \oplus z$ is defined and using $\left(\alpha_{1}\right),\left(\gamma_{1}\right)$ and (iii) we have $\left(\left(x \oplus y^{-}\right) \oplus z^{-}\right)=\left(\left(x^{-} \oplus y\right) \oplus z\right)^{-}=\left(x^{-} \oplus(y \oplus z)\right)^{-}=$ $\left(x \oplus\left(y^{-} \oplus z^{-}\right)\right)$.

Case vii. Let $\left(x^{-} \oplus y\right) \oplus z^{-}$and $y \oplus z^{-}$be defined. Then $y^{-} \oplus z$ is defined and with $\left(\alpha_{1}\right),\left(\gamma_{1}\right)$ and (iv) we can see that $\left(\left(x^{-} \oplus y\right) \oplus z^{-}\right)=\left(\left(x \oplus y^{-}\right) \oplus z\right)^{-}=$ $\left(x \oplus\left(y^{-} \oplus z\right)\right)^{-}=\left(x^{-} \oplus\left(y \oplus z^{-}\right)\right)$.

Case viii. Let $\left(x^{-} \oplus y^{-}\right) \oplus z^{-}$and $y^{-} \oplus z^{-}$be defined. Then $y \oplus z$ is defined and $\left(x^{-} \oplus y^{-}\right) \oplus z^{-}=(x \oplus y)^{-} \oplus$ $z^{-}=((x \oplus y) \oplus z)^{-}=(x \oplus(y \oplus z))^{-}=x^{-} \oplus\left(y^{-} \oplus z^{-}\right)$. 
Hence $(G, \oplus, 0)$ forms an a-commutative partial group. Since $E$ is a generalized effect algebra, by Lemma 7 there exists a relation $\leq^{G}$ such that $(G, \oplus, 0)$ w.r.t. $\leq G$ forms a woa-group.

Example 2. An interval $[-1,1]$ with $\oplus$ defined for $0 \leq x, y$ by

- $x \oplus y=x+y$ iff $x+y \leq 1$,

- $x \oplus(-y)=x-y$,

- $(-x) \oplus(-y)=-(x \oplus y)$ iff $(x \oplus y)$ exists

and relation $\leq^{G}$ defined by $x \leq^{G} y$ iff $x \leq y$ and $y-x \leq 1$ for all $x, y \in[-1,1]$ forms a woa-group. A positive cone $([0,1], \oplus /[0,1], 0)$ forms a well-known unit interval effect algebra.

\section{Hilbert spaces}

We assume that $\mathcal{H}$ is an infinite-dimensional complex Hilbert space, i.e., a linear space with inner product $\langle\cdot, \cdot\rangle$ which is complete in the induced metric. The term dimension of $\mathcal{H}$ in the following always means the Hilbertian dimension defined as the cardinality of any orthonormal basis of $\mathcal{H}$ (see [1]).

Moreover, we will assume that all considered linear operators $A$ (i.e., linear maps $A: D(A) \rightarrow \mathcal{H}$ ) have a domain $D(A)$ a linear subspace dense in $\mathcal{H}$ with respect to the metric topology induced by the inner product, so $\overline{D(A)}=\mathcal{H}$ (we say that $A$ is densely defined). We denote by $\mathcal{D}$ the set of all dense linear subspaces of $\mathcal{H}$. By positive linear operators $A$, (denoted by $A \geq 0$ ) it means that $\langle A x, x\rangle \geq 0$ for all $x \in D(A)$.

To every linear operator $A: D(A) \rightarrow \mathcal{H}$ with $\overline{D(A)}=\mathcal{H}$ there exists the adjoint operator $A^{*}$ of $A$ such that $D\left(A^{*}\right)=\left\{y \in \mathcal{H} \mid\right.$ there exists $y^{*} \in \mathcal{H}$ such that $\left\langle y^{*}, x\right\rangle=\langle y, A x\rangle$ for every $\left.x \in D(A)\right\}$ and $A^{*} y=y^{*}$ for every $y \in D\left(A^{*}\right)$. When $A^{*}=A, A$ is called self-adjoint (for more details see [1]).

Recall that a linear operator $A: D(A) \rightarrow \mathcal{H}$ is called a bounded operator if there exists a real constant $C \geq 0$ such that $\|A x\| \leq C\|x\|$ for all $x \in D(A)$ and hence $A$ is an unbounded operator if to every $C \in \mathbb{R}, C \geq 0$ there exists $x_{C} \in D(A)$ with $\left\|A x_{C}\right\|>C\left\|x_{C}\right\|$. By symbol 0 we mean a null operator and it is a bounded operator. The set of all bounded operators on $\mathcal{H}$ is denoted by $\mathcal{B}(\mathcal{H})$. For every bounded operator $A: D(A) \rightarrow \mathcal{H}$ densely defined on $D(A)=D \subset \mathcal{H}$ exists a unique extension $B$ such as $D(B)=\mathcal{H}$ and $A x=B x$ for every $x \in D(A)$. We will denote this extension $B=A^{\mathbf{b}}$ (see again [1]).

Definition 5. For an infinite-dimensional complex Hilbert space $\mathcal{H}$, let us define these sets of operators (in order to [4, 6]):

- $\mathcal{G} r(\mathcal{H})=\{A: D(A) \rightarrow \mathcal{H} \mid \overline{D(A)}=\mathcal{H}$ and $D(A)=$ $\mathcal{H}$ if $A$ is bounded $\}$

- $\mathcal{G} r_{D}(\mathcal{H})=\{A \in \mathcal{G} r(\mathcal{H}) \mid D(A)=D$ or $A$ is bounded\}
- $\mathcal{S} a \mathcal{G} r(\mathcal{H})=\left\{A \in \mathcal{G} r(\mathcal{H}) \mid A=A^{*}\right\}$

- $\mathcal{S} a \mathcal{G} r_{D}(\mathcal{H})=\{A \in \mathcal{S} a \mathcal{G} r(\mathcal{H}) \mid D(A)=D$ or $A$ is bounded $\}$

- $\mathcal{V}(\mathcal{H})=\{A \in \mathcal{G} r(\mathcal{H}) \mid A \geq \mathbf{0}\}$.

We also define an operation $\oplus_{\mathcal{D}}$ on $\mathcal{G} r(\mathcal{H})$ by

$A \oplus_{\mathcal{D}} B= \begin{cases}A+B & \text { if } A+B \text { is unbounded and } \\ & (D(A)=D(B) \text { or } \\ & \text { one out of } A, B \text { is bounded }), \\ (A+B)^{\mathbf{b}} & \text { if } A+B \text { is bounded } \\ & \text { and } D(A)=D(B), \\ \text { undefined } & \text { otherwise, }\end{cases}$

and operation $\oplus_{\mathbf{u}}$ by $A \oplus_{\mathbf{u}} B=A \oplus_{\mathcal{D}} B$ iff at least one of $A, B \in \mathcal{G} r(\mathcal{H})$ is bounded or $A, B \in \mathcal{G} r(\mathcal{H})$ are both unbounded, $D(A)=D(B)$ and there exists a real number $\lambda_{B}^{A} \neq 0$ such that $A-\lambda_{B}^{A} B$ is bounded[4].

For an arbitrary subset $X \subseteq \mathcal{G} r(\mathcal{H})$ let us define a relation $\leq_{\mathcal{D}}^{X}\left(\right.$ resp. $\left.\leq_{\mathbf{u}}^{X}\right)$ such that for any $A, B \in X$, $A \leq_{\mathcal{D}}^{X} B\left(\right.$ resp. $\left.A \leq_{\mathbf{u}}^{X} B\right)$ iff there exists a positive $C \in X$ such that $A \oplus_{\mathcal{D}} C=B$ (resp. $A \oplus_{\mathbf{u}} C=B$ ).

Theorem 3. Let $\mathcal{H}$ be an infinite-dimensional complex Hilbert space. Then $\left(\mathcal{G} r(\mathcal{H}), \oplus_{\mathcal{D}}, \mathbf{0}\right)$ w.r.t. relation $\leq_{\mathcal{D}}^{\mathcal{G} r(\mathcal{H})}$ forms a woa-group. Moreover, $\left(\mathcal{G} r_{D}(\mathcal{H}), \oplus_{\mathcal{D} / \mathcal{G} r_{D}(\mathcal{H})}, \mathbf{0}\right)$ w.r.t. relation $\leq_{\mathcal{D}}^{\mathcal{G} r_{D}(\mathcal{H})}$ forms its woa-subgroup.

Proof. It has been shown [6] that $\left(\mathcal{G} r(\mathcal{H}), \oplus_{\mathcal{D}}, \mathbf{0}\right)$ w.r.t. relation $\leq_{\mathcal{D}}^{\mathcal{G} r(\mathcal{H})}$ forms a wop-group. Moreover, in [4, Lemma 4] the axiom (Giv) is proved. Axiom (Ri) holds by definition. Since $\left(\mathcal{V}(\mathcal{H}), \oplus_{\mathcal{D}} / \mathcal{V}(\mathcal{H}), \mathbf{0}\right)=$ $\left(\operatorname{Pos}(\mathcal{G} r(\mathcal{H})), \oplus_{\mathcal{D} / \operatorname{Pos}(\mathcal{G} r(\mathcal{H}))}, \mathbf{0}\right)$ is a generalized effect algebra 8, (Rii) and (Riii) hold. According to [6] $\left(\mathcal{G} r_{D}(\mathcal{H}), \oplus_{\left.\mathcal{D} / \mathcal{G} r_{D}(\mathcal{H}), 0\right)}\right.$ w.r.t. $\leq_{\mathcal{D}}^{\mathcal{G} r(\mathcal{H})}$ is a wopsubgroup hence by Corollary 1 it is also a woasubgroup.

Note that since the operation $\oplus_{\mathcal{D} / \mathcal{G} r_{D}(\mathcal{H})}$ is total on $\mathcal{G} r_{D}(\mathcal{H})$, it is also a partially ordered commutative group.

Theorem 4. Let $\mathcal{H}$ be an infinite-dimensional complex Hilbert space. Then $\left(\mathcal{G} r(\mathcal{H}), \oplus_{\mathbf{u}}, \mathbf{0}\right)$ w.r.t. relation $\leq_{\mathbf{u}}^{\mathcal{G} r(\mathcal{H})}$ forms a woa-group. Moreover, $\left(\mathcal{G} r_{D}(\mathcal{H}), \oplus_{\mathbf{u} / \mathcal{G} \mathbf{r}_{\mathbf{D}}(\mathcal{H})}, \mathbf{0}\right)$ w.r.t. relation $\leq_{\mathbf{u}}^{\mathcal{G} r_{D}(\mathcal{H})}$,

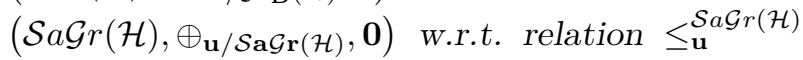
and $\left(\mathcal{S} a \mathcal{G} r_{D}(\mathcal{H}), \oplus_{\mathbf{u}} / \mathcal{S} \mathbf{a G} \mathbf{r}_{\mathbf{D}}(\mathcal{H}), \mathbf{0}\right)$ w.r.t. relation $\leq_{\mathbf{u}} \mathcal{S a G r}_{D}(\mathcal{H})$ form its woa-subgroups.

Proof. We have shown [6] that $\left(\mathcal{G} r(\mathcal{H}), \oplus_{\mathbf{u}}, \mathbf{0}\right)$ w.r.t. relation $\leq \mathbf{G} r(\mathcal{H})$ forms a wop-group. In [4, Lemma 6]. is proved the axiom (Giv). (Ri) holds by definition. Because $\left(\mathcal{V}(\mathcal{H}) \oplus_{\mathbf{u}} \mathcal{V}(\mathcal{H}), \mathbf{0}\right)=$ $\left(\operatorname{Pos}(\mathcal{G r}(\mathcal{H})), \oplus_{\mathbf{u} / \operatorname{Pos}(\mathcal{G} r(\mathcal{H}))}, \mathbf{0}\right)$ is a generalized effect algebra [4, 7], we have (Rii) and (Riii). According to

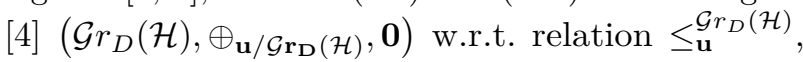




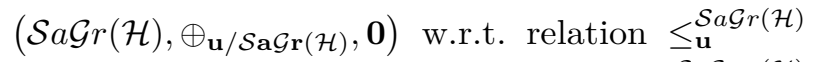

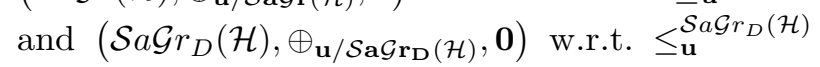
are wop-subgroups hence by using Corollary 1 they are woa-subgroups.

\section{ACKNOWLEDGEMENTS}

The author gratefully acknowledges financial support from Masaryk University under grant 0964/2009 and financial support from ESF Project CZ.1.07/2.3.00/20.0051 Algebraic methods in Quantum Logic of the Masaryk University.

\section{REFERENCES}

[1] Blank J., Exner P., Havlíček M., Hilbert Space Operators in Quantum Physics, 2nd edn. Springer, Berlin (2008).

[2] Dvurečenskij A., Pulmannová S., New Trends in Quantum Structures, Kluwer Acad. Publ.,

Dordrecht/Ister Science, Bratislava, 2000.
[3] Foulis D. J., Bennett M. K., Effect algebras and unsharp quantum logics, Found. Phys. 24 (1994), 1331-1352.

[4] Janda J., Weakly ordered partial commutative group of self-adjoint operators densely defined on Hilbert space, Tatra Mt. Math. Publ., 50 (2011), 1-16.

[5] Paseka J., $\mathcal{P} \mathcal{T}$-Symmetry in (Generalized) Effect Algebras, Internat. J. Theoret. Phys., 50 (2011), 1198-1205.

[6] Paseka J., Janda J., More on $\mathcal{P} \mathcal{T}$-Symmetry in (Generalized) Effect Algebras and Partial Groups, Acta Polytechnica, 51 (2011), No. 4, 65-72.

[7] Paseka J., Riečanová Z., Considerable Sets of Linear Operators in Hilbert Spaces as Generalized Effect Algebras. Found. Phys. 41 (2011), 1634-1647.

[8] Riečanová Z., Zajac M. and Pulmannová S., Effect Algebras of Positive Linear Operators Densely Defined on Hilbert Spaces, Reports on Mathematical Physics 68, (2011), 261-270. 